\title{
A Theoretical Study of Broadband Extraordinary Optical Transmission in Gold Plasmonic Square Nanohole Arrays and its Application on Refractive Index Sensor
}

\author{
Rehan Shafiq \\ Sarhad University: Sarhad University of Science \& Information Technology \\ Javed lqbal \\ Sarhad University: Sarhad University of Science \& Information Technology \\ Adnan Daud Khan \\ UET Peshawar: University of Engineering \& Technology \\ Anees Ur Rehman ( $\square$ anees.ee@suit.edu.pk) \\ Sarhad University of Science and Information Technology Ring Road Campus
}

\section{Research Article}

Keywords: Extraordinary optical transmission, plasmonic square nanoholes, periodic disorder, refractive index sensing

Posted Date: September 8th, 2021

DOI: https://doi.org/10.21203/rs.3.rs-852168/v1

License: (9) This work is licensed under a Creative Commons Attribution 4.0 International License. Read Full License

Version of Record: A version of this preprint was published at Optical and Quantum Electronics on February 11th, 2022. See the published version at https://doi.org/10.1007/s11082-022-03519-9. 


\title{
A Theoretical Study of Broadband Extraordinary Optical Transmission in Gold Plasmonic Square Nanohole Arrays and its Application on Refractive Index Sensor
}

\author{
Rehan Shafiq ${ }^{1 *}$, Javed Iqbal ${ }^{1}$, Adnan Daud Khan ${ }^{2}$, Anees Ur Rehman ${ }^{1 * *}$ \\ ${ }^{1}$ Department of Electrical Engineering, Sarhad University of Science and Information Technology, Peshawar 25220, \\ Khyber Pukhtunkhwa, Pakistan \\ ${ }^{2}$ Center for Advanced Studies in Energy, University of Engineering and Technology, Peshawar 25124, Khyber \\ Pukhtunkhwa, Pakistan \\ Corresponding Authors: *rehanshafiq84@gmail.com; **anees.ee@ suit.edu.pk
}




\begin{abstract}
Extraordinary optical transmission (EOT) behaviour is investigated in a subwavelength plasmonic nanostructure, consisting of a gold film perforated with a square nanohole array and deposited on a silicon dioxide substrate. The essential aspect of the proposed structure is the periodic disorder that enables broadband transmission peaks in the visible and near-infrared region and reduces the structure's size, which mainly arises from the excitation of localized surface plasmon resonances (LSPRs). Optical cavity modes formed in the nanoholes and the hybridization of plasmon modes. Further, the performance of the proposed nanostructure as a plasmonic sensor is analyzed by increasing the index of refraction of the local environment; the EOT exhibit remarkable refractive index sensitivity of up to $944 \mathrm{~nm} / \mathrm{RIU}$, a figure of merit of 9.25 and a contrast ratio of $47 \%$ are realized. The proposed structure has some practical significance for designing low-cost and effective sensing devices.
\end{abstract}

Keywords: Extraordinary optical transmission; plasmonic square nanoholes; periodic disorder; refractive index sensing. 


\section{Introduction}

Extraordinary optical transmission (EOT) was first studied in a thin metallic film by T.W Ebbessen in 1998. He found that when the aperture size is smaller than the wavelength of light, it enhances light transmission [1]. Since its discovery, it has gained significant attention among researchers because of its potential applications in many fields, like optoelectronics [2], biological sensors [3], surface-enhanced Raman scattering [4].

In metallic nanosystems, plasmon oscillations arise due to the interaction of electromagnetic fields and the conduction electrons in a metal, propagating along the metal surface. These propagating surface plasmons are known as surface plasmon polaritons (SPPs), which can overcome the conventional diffraction limit and manipulate the propagation of light on the subwavelength scale [5-7]. When periodic holes are incorporated into a metal structure, these SPPs enhance light transmission [2]. It is also found that the localized surface plasmon resonances (LSPRs) arising from the individual resonances of the plasmonic nanoparticles or apertures can also contribute to the EOT phenomenon [8]. The coupling between SPPs and LSPRs resulting in extraordinary optical behaviours like EOT[9-12]. The behaviour of EOT strongly depends on shapes, sizes, hole arrangements, and dielectrics [13-17], which provide us with the opportunity to design novel plasmonic nanostructures to manipulate light at the nanoscale.

Many researchers have experimentally investigated the EOT effect in perforated metallic nanostructures. For example, Ekinci et al. have analyzed EOT behaviour in the ultraviolet (UV) range in aluminium nanohole arrays [18]. Najiminaini et al. constructed a 3D plasmonic nanostructure containing an array of free-standing gold nanohole film above a cavity with a uniform depth and a cluster of gold nanodisks at the bottom of the cavity associated with the 
nanohole array [19]. The proposed architecture supports EOT by coupling localized surface plasmon between nanodisks and nanoholes. They also showed that the LSPR peak position depends on the nanohole and nanodisk diameter and differentiation between them. Further, they found that the LSPR binding between each nanohole and the corresponding nanodisk virtually vanished for small cavity depth values, and highest transmission peak was observed for the lowest cavity.

The configuration of the Fabry-Perot Resonant Cavity, with both ends of it open, has been proposed by Wang et al., who used a number of X-shape nanoholes in a silver film [20]. Clear propagation peaks in the spectrum, which occur on the top surface of the silver film due to SPPs, the Fabry-Perot resonance effect of SPPs in the nanohole, and the nanohole LSPR, were observed. The transmitting properties of the proposed design depend on structural parameters such as nanohole diameter and silver film thickness, and the incident field polarization, which could be ideal for the design of a tunable plasmonic filter.

Zhang et al. have reported a plasmonic nanostructure composed of a silver film perforated with a 2D square array of two-layer cylindrical nanoholes based on a silica substrate [9]. Recently, Hu et al. demonstrated a subwavelength plasmonic nanostructure composed of a gold film perforated with a slot antenna array that is connected. The highest transmission intensity of more than $90 \%$ is attained via the LSPR at the edges of nanoholes, the SPPs on the surface of the gold film, and Fabry-Férot resonances of surface plasmons in the nanoholes. Shinpei et al. propose a hexagonal boron nitride (hbn) structure. They show that lspr peaks position depends on the nanohole and nanodisk diameter[21]. Yu Lin et al. demonstrate a plasmonic structure composed of the silver metallic film. This work offers a way to adjust the transmission peak's red or blue shift and localize resonant performance at a particular frequency[22]. Shaziyeh Tajik et al. analyzed EOT 
in Multilayer circular hole array[23]. Jongkyoon park et al. constructed the bowtie structure studied the gap sizes and analyzed transmission intensities[24].

However, all the structures reported in the literature based on EOT are symmetric structures; although they achieve high transmission intensities, no attention has been focused on structure size. In simple words, to achieve high transmission, the designer must increase the hole or cavity size, increasing the structure dimensions. In this work, we suggest ideas leading to achieve EOT by introducing an asymmetric structure. Based on the author's understanding, asymmetric structure to achieve broad mode EOT has not previously been thoroughly addressed. This observation can also help reduce the size of the device. In order to obtain these EOT observations, we theoretically investigated a thin metallic gold film perforated with square nanoholes arrays both symmetrically and asymmetrically based on the silicon dioxide substrate. The optical properties of our proposed structure and electric field intensity distribution were calculated by the three-dimensional finite element method. It is found that highly enhanced transmission with maximum transmittance over $93 \%$ is obtained due to the excitation and near field coupling of strong LSPRs, SPPs, and the gap plasmon modes supported by holes. The position and intensity of the broad EOT peak can be effectively tuned by changing the structure parameters because of their effect on the distribution of oscillating charges on metal surfaces. the highly tunable EOT phenomenon in our proposed structure has potential in refractive index sensor to achieve maximum sensitivity values, the figure of merit and contrast ratio.

\section{Model}

The proposed design of the perforated square-shaped nanostructure is shown in Figure 1. The structure is composed of two layers; the upper gold and the bottom silicon dioxide substrate. The thickness of both layers is kept constant at 50nm. A periodic array of square nanoholes are made 
in the gold layer to increase the transmission of incoming photons. The bottom layer will further improve the light after the top layer enhancement. Figure 1(b) shows a single unit cell structure consisting of square holes having dimensions of $\mathrm{L}=\mathrm{W}=80 \mathrm{~nm}$, where $\mathrm{L}$ is the length of the square hole, and W is the Width of the square hole. The size of the unit cell is $\mathrm{P}_{\mathrm{x}}=\mathrm{P}_{\mathrm{y}}=280 \mathrm{~nm}$ and is repeated along $\mathrm{x}$ and $\mathrm{y}$-directions due to periodic boundary conditions. The dielectric constant of gold is taken from Johnson and Christy data model [25], while the dielectric constant for $\mathrm{SiO}_{2}$ is taken as 3.9 [26]. The medium around the structure is air, and all the simulations are carried out in Comsol Multiphysics Software using the finite element method (FEM). The simulation uses an excellent mesh control system, and the mesh chooses free tetrahedral. The maximum and minimum cell size is 20 and $.04 \mathrm{~nm}$. The polarized light energized the waveguide, and SPPs travelled through the waveguide. In order to determine the structure's electromagnetic wave transmission spectrum, input and output ports corresponding to $\mathrm{P}_{1}$ and $\mathrm{P}_{2}$ incident power are detected. In addition, the shape in the nanoscale scale allows better use in plasmonic devices.

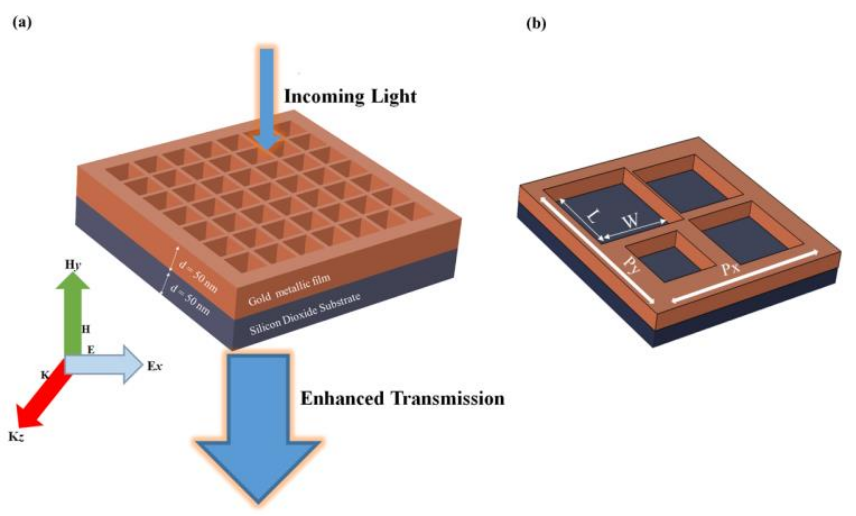

Figure 1(a). Schematic view of perforated square nanohole structure. (b) A unit cell of the structure containing Asymmetric square nanoholes.

\section{Results and Discussions}

Since EOT bands can be efficiently tailored by varying the nanostructure's size, shape, and material, we added periodic disorders/defects inside the unit cell by varying the size of each nanohole. In the square lattice of nanoholes, several combinations of defects can be classified into four categories. The first 
classification is the case without defect (symmetric); the sizes of each nanohole are equivalent. Additional categories include one, two, and three defects (asymmetric) in the unit cell, indicating different sizes of the nanoholes.

In the first case, we considered a simple symmetric square nanohole array (SNA), where all the sizes of the holes are identical, each having a size of $80 \mathrm{~nm}$, i.e., $S=\left[\begin{array}{ll}80 & 80 \\ 80 & 80\end{array}\right]$. Then, we computed the transmission spectra of the symmetric structure for polarization, i.e., along the $x$-axis, as shown in Figure. 2(a). The red curve represents the polarization along the $x$-axis, where the two EOT bands are found at $510 \mathrm{~nm}$ and $680 \mathrm{~nm}$ wavelengths with peak intensities of $60 \%$ and $80 \%$, respectively. These modes are obtained by hybridizing plasmon modes, LSPRs, and the optical cavity mode [16-19].

The electric field distributions are also computed at respective transmission peaks, as shown in Figure 2(b). At 510nm, the electric field is confined at the edges of the nanoholes, which indicates the excitation and hybridization of low wavelength plasmon modes. At $685 \mathrm{~nm}$, the strong electric field is confined at the edges of the holes and specifies the excitation and hybridization of high wavelength plasmon modes. A small number of electric field distributions can be seen at some corners, demonstrating the existence of dark mode that has not appeared in the spectrum due to spectrally narrowed and weakly damped. This mode can be excited by symmetry breaking or adding defects in the lattice.

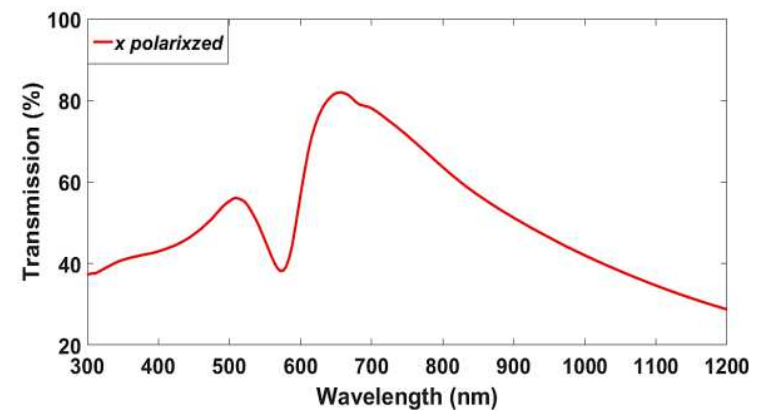

(a)

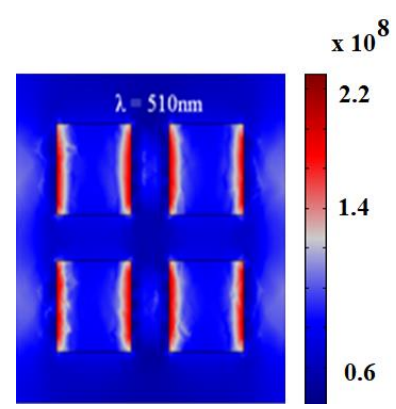

(b)

Figure 2(a). Calculated Transmission spectra for symmetric case. (b) Electric field distributions at resonant peaks.

In the second case, we investigated the single defective SNA where the size of one nanohole is changed. Thus, we have a set of two different sizes in a lattice. We selected unique combinations, i.e., S1= 
$\left[\begin{array}{cc}80 & 80 \\ 104 & 80\end{array}\right], S 2=\left[\begin{array}{cc}80 & 80 \\ 104 & 104\end{array}\right], S 3=\left[\begin{array}{cc}104 & 104 \\ 104 & 80\end{array}\right]$, and $S 4=\left[\begin{array}{cc}80 & 104 \\ 104 & 80\end{array}\right]$ (the matrix represents the sizes of the nanoholes in a lattice). Figure 3(a) shows the optical transmission characteristics of S1, S2, S3, and S4 structures. For S1 (red curve), three transmission peaks are observed at 520nm, 680nm, and 1000nm, respectively. The broad mode at $680 \mathrm{~nm}$ has sustained its spectral position while the transmission intensity is increased from $82 \%$ to $89 \%$ due to symmetry breaking. The short transmission peak at $520 \mathrm{~nm}$ has slightly changed its spectral location, and the transmission intensity is also marginally improved by comparing with the symmetric case. A new transmission peak at $1000 \mathrm{~nm}$ appears, which was dark in the symmetric structure. This peak arises due to symmetry breaking where modes of different angular momentum mix strongly [27].

For the S2 structure (yellow curve), the resonances obtained are almost similar to the symmetric case except that the transmission intensities are improved. The transmission spectra for the structure S3 are indicated by a blue curve where four resonant modes are obtained at $520 \mathrm{~nm}, 680 \mathrm{~nm}, 920 \mathrm{~nm}$, and 1140nm, and their peak amplitudes are increased compared to S1 and S2 cases. This configuration is considered to be the best among others due to an enhanced transmission. S4 (green) response is similar to S2, except for a short resonant mode occurring at 910nm due to the position of the defects.

According to the results, the transmission intensity increased, but the broad mode is also attained in S3. As a result, $\mathrm{S} 3$ is our preferred option; in $\mathrm{S} 3$, a strong resonant peak centered at $680 \mathrm{~nm}$ is achieved, with maximum transmission up to $89 \%$ and full width at half maximum (FWHM) up to $220 \mathrm{~nm}$.

We computed the electric field distribution for a single defective structure at respective peaks as shown in Figure 4 (a-m). Figure 4 (a-c) shows the electric field distribution for S1.at 510nm; the electric field is confined at the edges of the nanoholes, which indicates the excitation and hybridization of low wavelength plasmon modes. At $685 \mathrm{~nm}$ strong and uniform electric field is confined at the edges of the holes, and strong electric field energy occurs in the center of the holes, illustrating the LSPR modes propagating in the holes[28]. At $862 \mathrm{~nm}$, an electric field is confined at the edges of the two holes, which specifies the excitation and hybridization of high wavelength plasmon modes which arises due to 
asymmetric structure, which is dark in symmetric structure. Figure 4(d-e) shows the electric field distribution for $\mathrm{S} 2$ at wavelength $515 \mathrm{~nm}$ and670nm. At 670nm strong electric field occur at the edges and center of the holes. Figure 4(f-i) exhibits the electric field distribution for S3 at wavelength 519nm, $685 \mathrm{~nm}$, and $1130 \mathrm{~nm}$. Due to asymmetric high wavelength transmission peaks are appeared at 900 and $1130 \mathrm{~nm}$ strong, and a uniform electric field occurred at $685 \mathrm{~nm}$ prominent transmission peak. Figure 4(j-1) shows the S4 electric field distribution at respective peaks where prominent electric field appeared at $690 \mathrm{~nm}$, and dark mode appeared at $900 \mathrm{~nm}$.

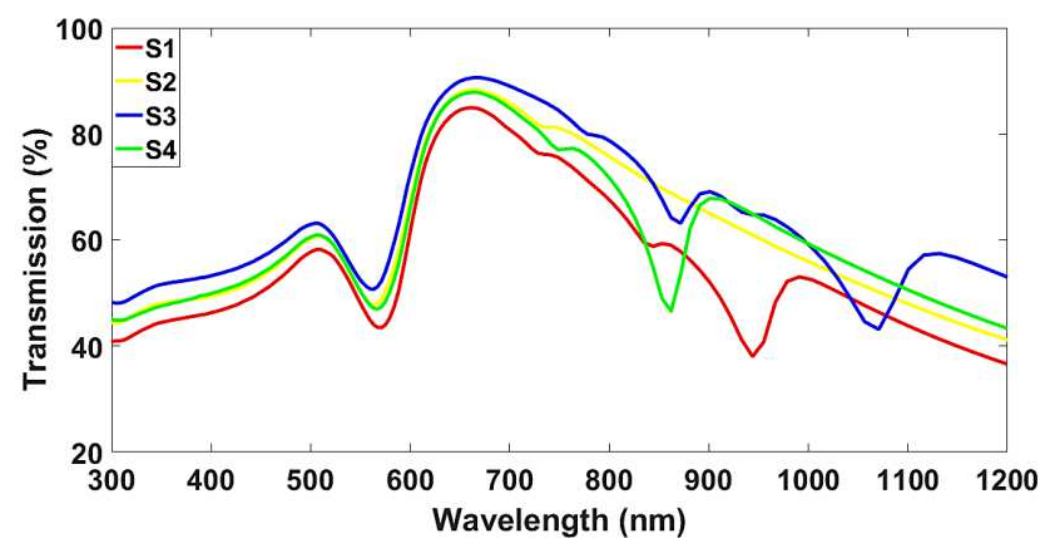

Figure 3. Calculated Transmission spectra for single defective structures S1, S2, S3, and S4. 


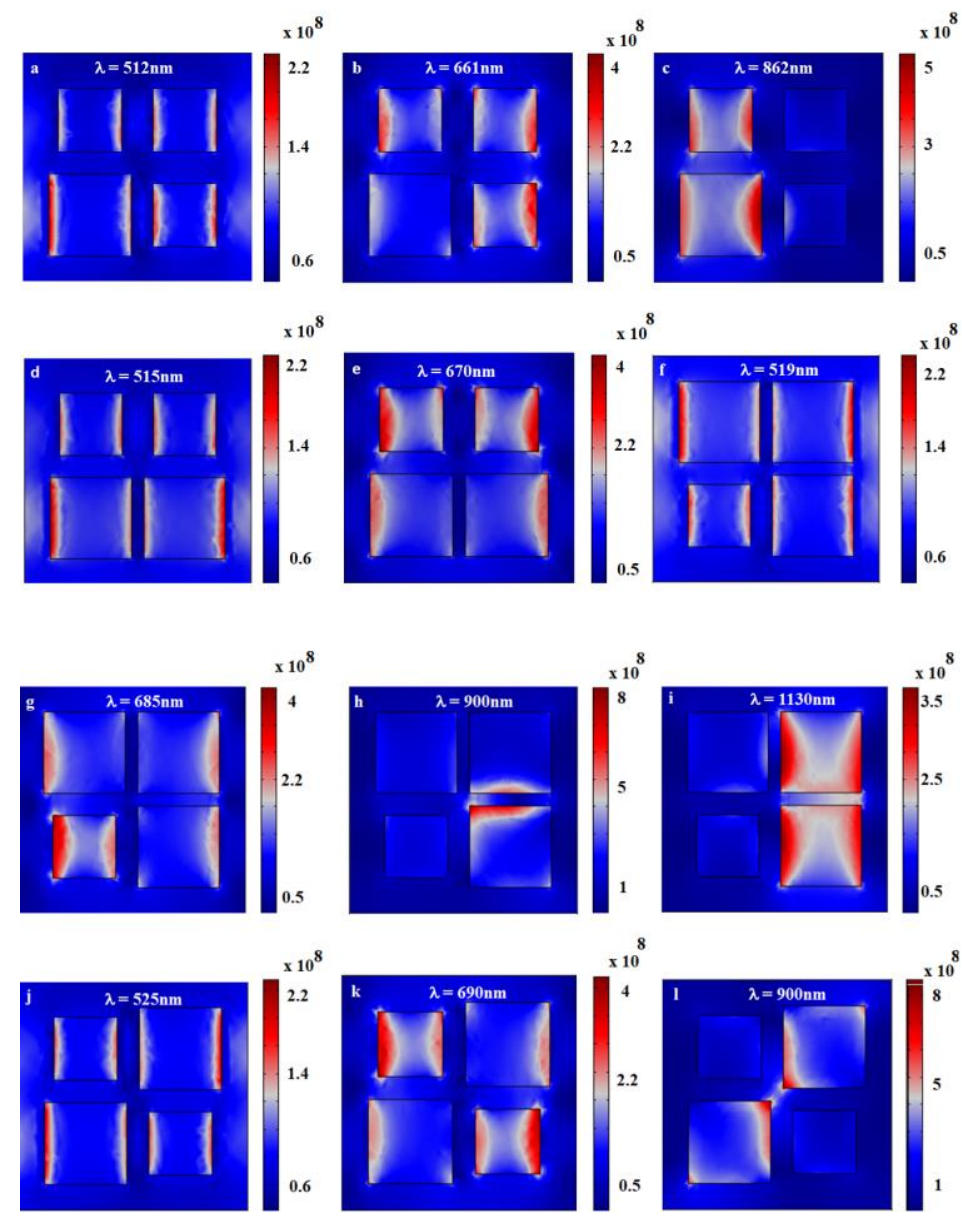

Figure 4(a-1) Electric field distributions of the proposed single defective structure at resonant peaks

Next, we introduced a double defective SNA structure where the sizes of two nanoholes are changed simultaneously in a lattice. So, in this way, we have three different sizes in a lattice, i.e., $S 5=\left[\begin{array}{cc}80 & 128 \\ 104 & 80\end{array}\right], S 6=\left[\begin{array}{cc}104 & 128 \\ 80 & 104\end{array}\right], S 7=\left[\begin{array}{cc}128 & 104 \\ 80 & 104\end{array}\right]$, and $S 8=\left[\begin{array}{cc}80 & 80 \\ 128 & 104\end{array}\right]$. Figure 4 shows the transmission spectra of S5, S6, S7, and S8 nanostructures, where the response of S7 is somehow similar to S3. S5 and S6 also exhibit four transmission peaks, but the amplitudes of high wavelength modes are low. S7 represents the most suitable case in this structure since S7 accomplishes FWHM 260nm and 93\% intensified transmission.

Figure 6 shows the electric field distribution for double defective structures at respective peaks for S5, S6, S7and S8. Figure 6 (b,f,l) shows strong electric field pattern is observed, suggesting 
the efficient light coupling effect. The hole sizes and gaps between the holes influence EOT largely[29]. Figure (a,c,h, and k) shows the electric field distribution for low wavelength plasmon modes, and c,d, and $\mathrm{j}$ shows the electric field distribution for high wavelength plasmon modes dark in symmetric structure.

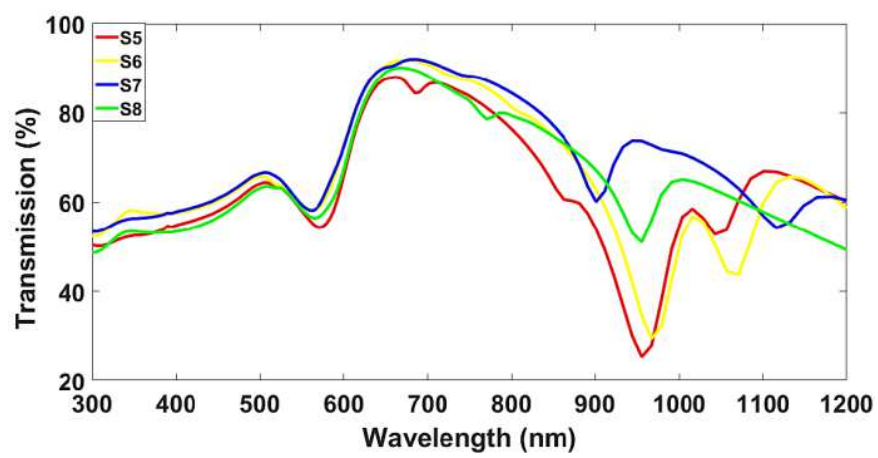

Figure 5. Calculated Transmission spectra for double defective structures S5, S6, S7, and S8. 


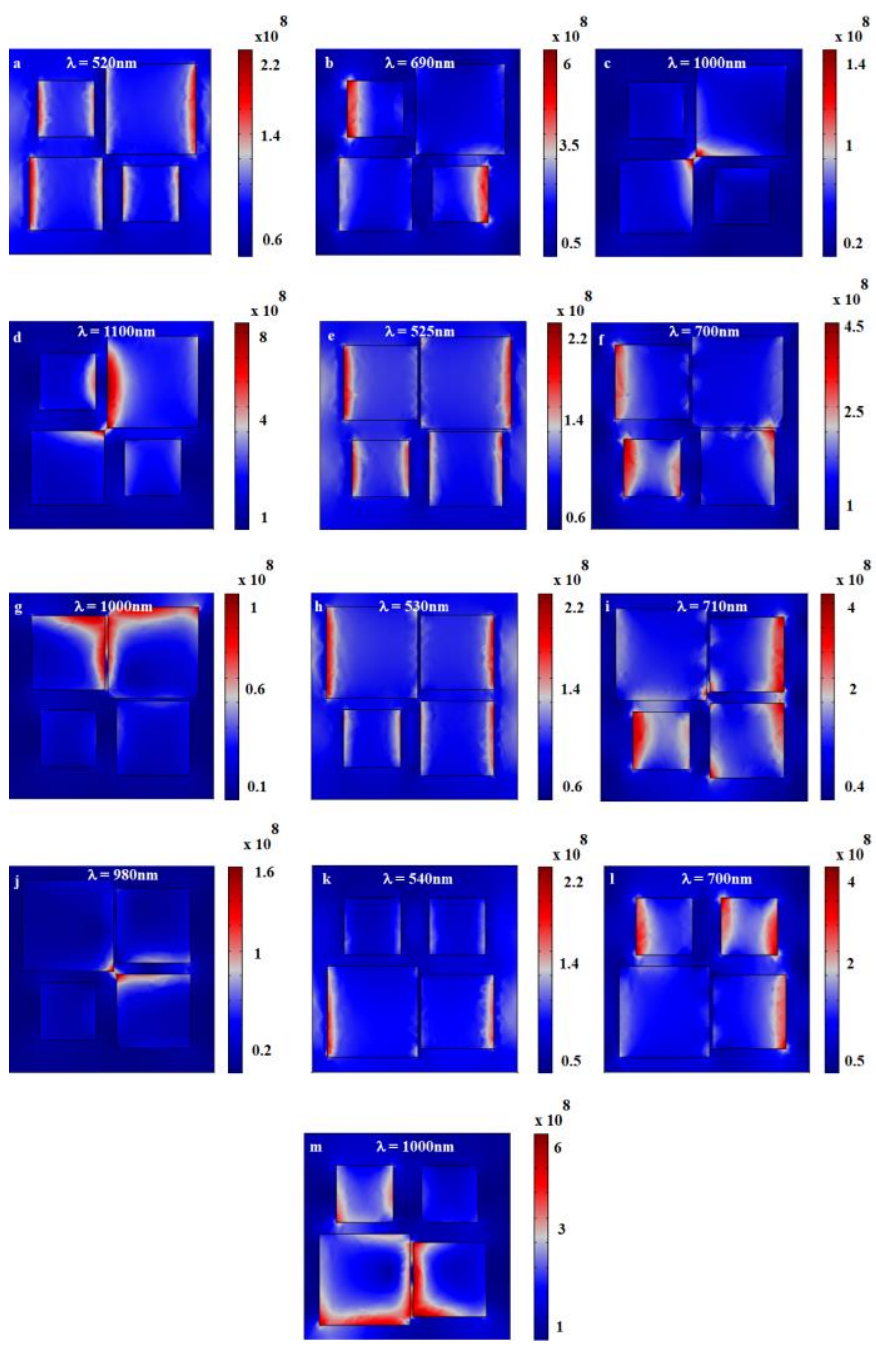

Figure 6 (a-m): Electric field distributions of the proposed double defective structure at resonant peaks

Finally, we introduced a triple defective SNA structure where the sizes of all the nanoholes are changed, i.e., $S 9=\left[\begin{array}{cc}132 & 104 \\ 104 & 80\end{array}\right]$ and $\mathrm{S} 10=\cdot\left[\begin{array}{cc}104 & 104 \\ 132 & 80\end{array}\right]$

Figure 5 shows the simulated spectra of a triple defective structure where both the structures exhibit high intensified and multiple peaks compared to a single and double defective structure. This is because the distance between the SNA is small, so, due to small distances, the surface plasmons interact strongly and interfere constructively; as a result, transmission is enhanced in a 
unit cell. Here, we achieved 97\% transmission and 300nm FWHM so, S10 is our preferred choice in triple defective structure.

Figure 8(a-g) calculated the electric field distribution at the corresponding resonant wavelengths observed in Figure 5. The primary field strengths at wavelength are limited to the holes' edges and centres, validating the excitation and coupling of LSPRs and SPPs. This results in enhanced transmission accompanied by broad bandwidth. Figure 8(c,f and g) depicts the weak electric field distribution for high wavelength localized at up and down holes, implying that LSPRs and SPPs interact weakly.

About the gap widths and thickness mentioned, it should be emphasized that, in proper experimental settings, such tiny gaps might lead to fabrication errors. Therefore, the minimal gap size in all the suggested structures was presumed to be $5 \mathrm{~nm}$ to have a topology that could be easily lifted in the fabrication process. It is a prevalent method that uses $5 \mathrm{~nm}$, and even less than $5 \mathrm{~nm}$ gap and thickness use from 50nm to $20 \mathrm{~nm}$ to construct plasmonic devices [28, 30-37]. The design of plasmonic filters included two linked resonators with $5 \mathrm{~nm}$ of coupling space [30]. The distance between the guides and resonators is similarly $5 \mathrm{~nm}$ [34]. Metallic structures with considerably less distance were utilized for developing sensors based on plasmonic filters [3234]. Compared to the structures proposed in this paper, such designs require a greater degree of fabrication accuracy. Based on past research, the suggested structure may have practical and suitable gap widths and thicknesses for the manufacturing process. 


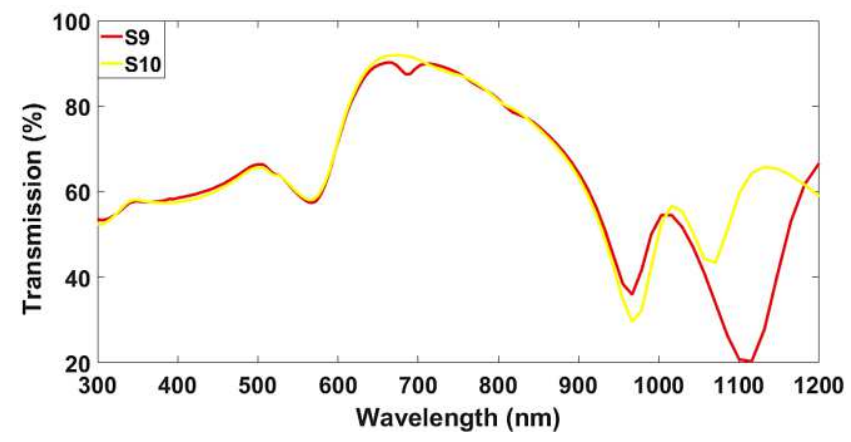

Figure 7: Calculated transmission spectra for triple defective structure.
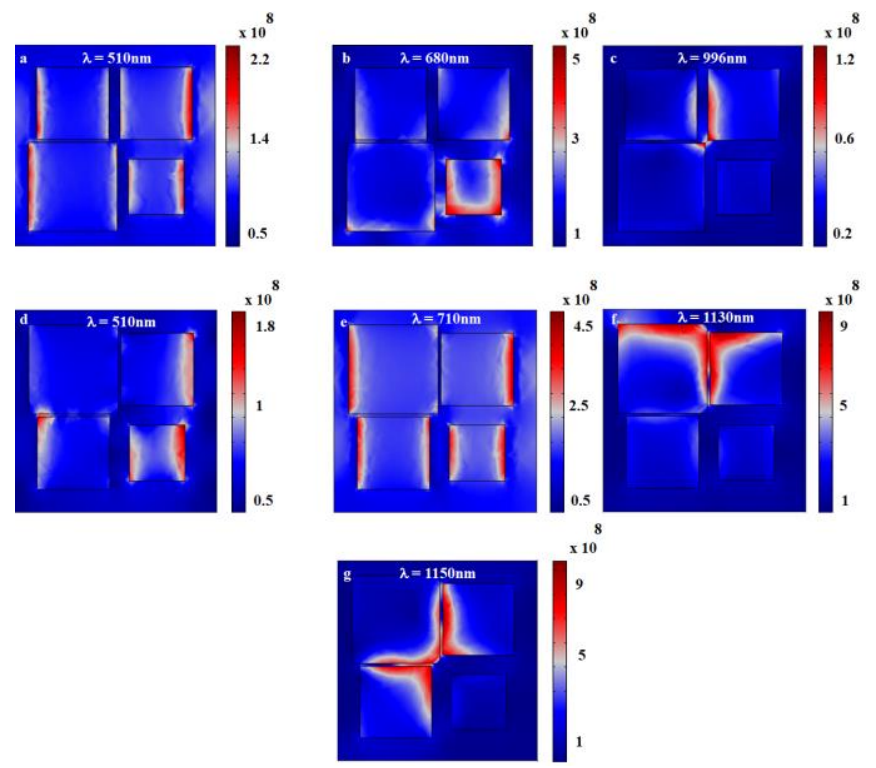

Figure 8 (a-g): Electric field distributions of the proposed triple defective structure at resonant peaks

\section{Sensing with SNA}

One of the essential applications for EOT resonances in plasmonic is sensors. To find out the sensing performance of our proposed structure, we took the best cases from all the configurations, i.e., S3 from a single defective structure, S7 from a double defective structure, and S9 from a triple defective structure, respectively. To analyze the efficiency of these EOT resonances as a biosensor, we use the Figure of Merits (FoM) and Contrast ratio (CR), which can be found from the different refractive indexes $(n)$ of the local environment [38-40]. The sensitivity is obtained by taking the ratio of change in wavelength and change in the refractive index of the medium, as shown by equation (1). The FoM is calculated by dividing the sensitivity 
over the bandwidth, as shown by equation (2). The CR is obtained by taking the ratio of difference and summation of peak and dip values of the resonant modes. The transmission spectra for various values of the embedding medium's refractive indices are shown in Figure 9(ac). There is a redshift and a modest rise in transmission intensity. The resonant wavelength positions for various refractive indices are shown in Figure 9(d-f). Various environmental parameters exhibit apparent spectrum shifts of modes. Table 1 compares the sensing performance of all peaks. The maximum values of sensitivity, FoM, and CR in two modes, ModeI and ModeII, for a single defective structure S3 are 444nm/RIU,4.44 and 47\%.

Similarly, for double defective structure S7, the maximum values of sensitivity, FoM, and CR in two modes, ModeI and ModeII, are 662nm/RIU,3.25 and 39\%. For triple defective structure S9, the maximum values of sensitivity, FoM, and CR in two modes, ModeI and ModeII 944nm/RIU,9.25 and 39\%. These results show that our proposed structure has some practical significance for designing effective sensing devices.

$$
\begin{gathered}
S=\frac{\lambda \max -\lambda \min }{n-1} \\
\boldsymbol{F} \boldsymbol{O M}=\frac{\boldsymbol{S}}{\boldsymbol{B}} \\
C R=\frac{(\text { Peak }- \text { dip }) \text { value }}{(\text { Peak }+ \text { dip }) \text { value }}
\end{gathered}
$$

(a)

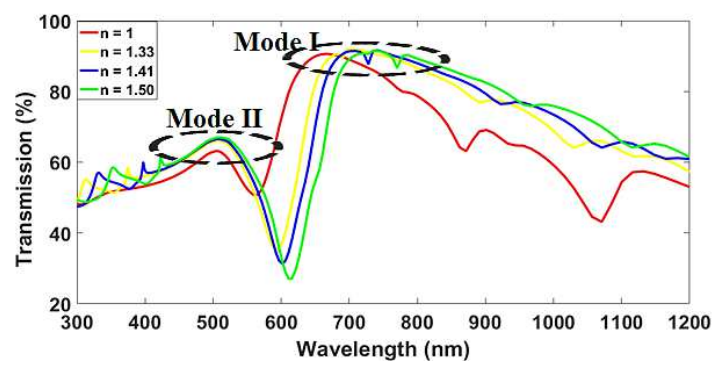

Single defective structure $\mathrm{S3}$

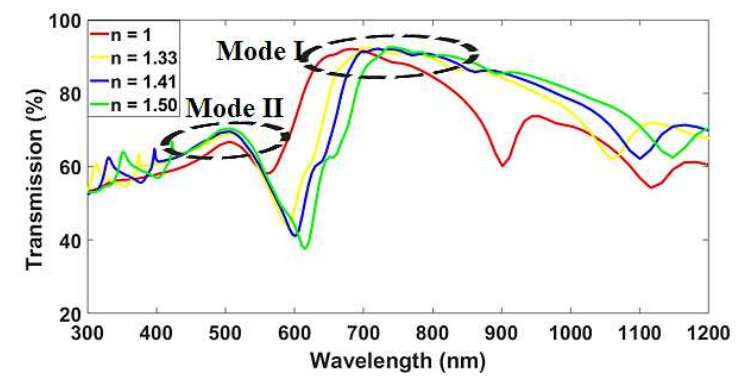


(c)

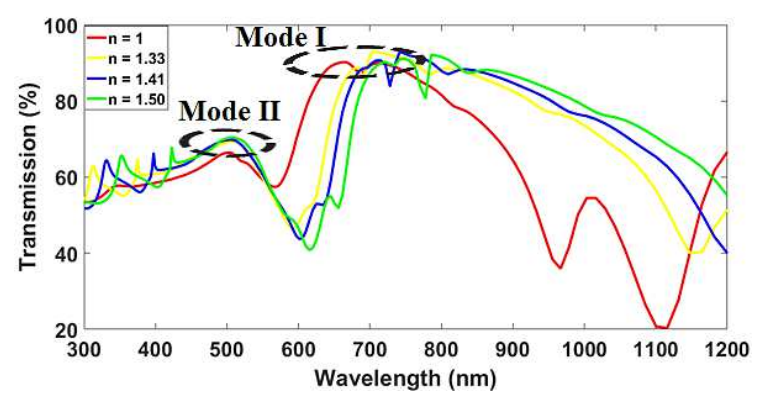

(e)

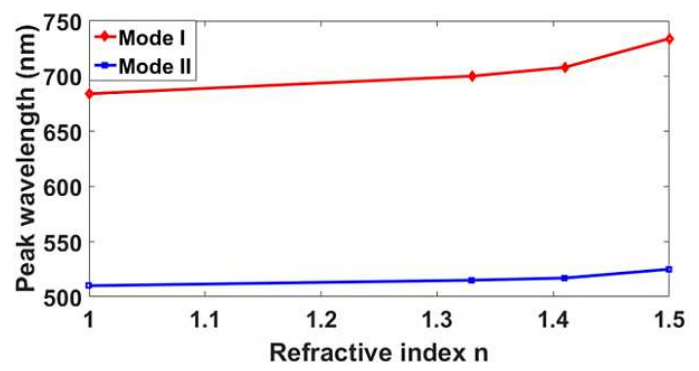

(d) Single defective structure S3

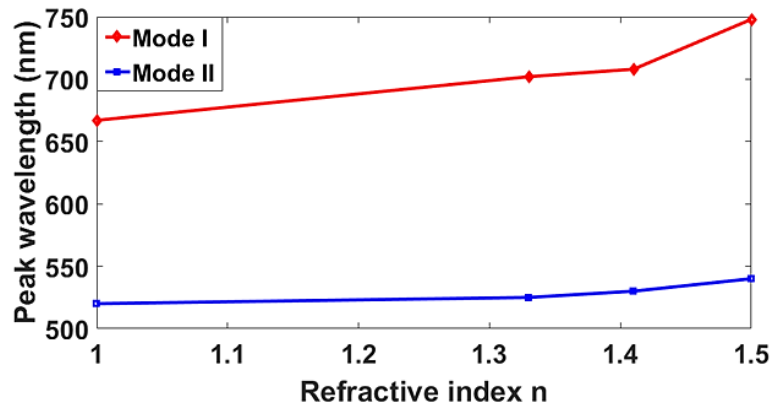

(f)

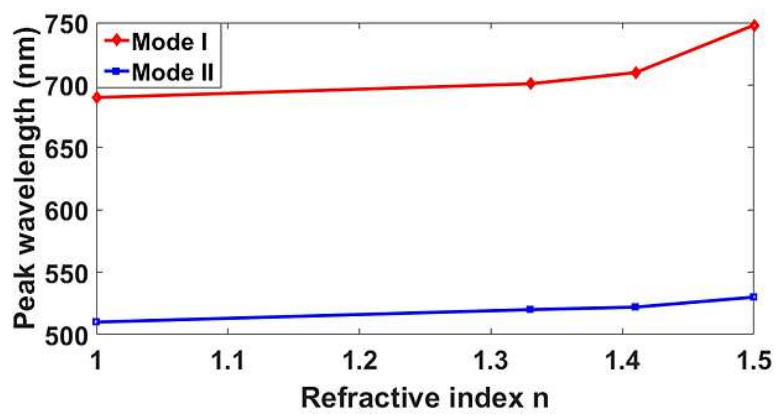

Figure 9: Transmission spectra versus refractive index for (a) single defective structure S3, (b) double defective structure S7, and (c) triple defective structure S10. (d-f) Pwavelength shifts of different modes as a function of refractive index.

Table 1. Sensitivity, FoM, and CR values for each mode supported by various refractive indices.

\begin{tabular}{|c|c|c|c|}
\hline Type & Sensitivitynm/RIU & FoM & CR (\%) \\
\hline & & & 29 \\
\hline \multirow{2}{*}{ Mode-I (Single Defective Structure S3) } & 162 & 1.9 & 44 \\
& 270 & 3.06 & 47 \\
\hline \multirow{2}{*}{ Mode-II (Single Defective Structure S3) } & 444 & 4.44 & 4.76 \\
\hline \multirow{2}{*}{ Mode-I (Double Defective Structure S7) } & 40 & .58 & 3 \\
\hline \multirow{2}{*}{ Mode-II (Double Defective Structure S7) } & 111 & 1.29 & 3.7 \\
\hline \multirow{2}{*}{ Mode-I (TRIPLE Defective Structure S10) } & 176 & 1.79 & 22 \\
& 352 & 1.62 & 31 \\
\hline \multirow{2}{*}{ Mode-II (Triple Defective Structure S10) } & 662 & 3.25 & 39 \\
\hline & 30 & .34 & 1.57 \\
& 58 & .55 & 2.9 \\
\hline & 544 & .96 & 2.1 \\
\hline
\end{tabular}




\section{Conclusions}

We numerically analyzed a metal nanostructure that consists of gold film perforated with a square nanohole array and deposited on a silicon dioxide substrate to achieve better transmission. The transmission spectrum of the structure in the visible and near-infrared wavelengths is calculated using the finite element approach. We also investigated the structure's defects. The calculations demonstrate that when defects are introduced to the surface of the metal film due to the interaction of SPPs and LSPRs, SNA can achieve EOT, and structure parameters have a significant influence on enhanced transmission. As a result, this SNA structure is significant in nanophotonic integration, nanolithography, and plasmon filters.

Furthermore, there is a linear relationship in the transmission spectrum between the resonant wavelength corresponding to the transmission peak and the refractive index $\mathrm{n}$. The peak wavelength increases as the refractive index $\mathrm{n}$ increases, and Mode $\mathrm{I}$ of the triple defective structure has a greater sensitivity of up to $944 \mathrm{~nm} / \mathrm{RIU}$. These findings have significant implications for the development of high sensitivity nanoscale refractive index sensors.

\section{Acknowledgment}

This work was financially supported by the board of advanced studies and research, Sarhad university of science and information technology, Peshawar Pakistan

\section{Conflicts of interest}

There are no conflicts to declare.

\section{References}

[1] T. W. Ebbesen, H. Lezec, H. F. Ghaemi, T. Thio, and P. A. Wolff, "Extraordinary Optical Transmission Through Sub-Wavelength Hole Arrays," Nature, vol. 391, pp. 667-669, 02/01 1998.

[2] W. L. Barnes, A. Dereux, and T. W. Ebbesen, "Surface plasmon subwavelength optics," (in eng), Nature, vol. 424, no. 6950, pp. 824-30, Aug 142003. 
[3] A. Dahlin, M. Zäch, T. Rindzevicius, M. Käll, D. S. Sutherland, and F. Höök, "Localized Surface Plasmon Resonance Sensing of Lipid-Membrane-Mediated Biorecognition Events," Journal of the American Chemical Society, vol. 127, no. 14, pp. 5043-5048, 2005/04/01 2005.

[4] L. Du, D. Tang, G. Yuan, S. Wei, and X. Yuan, "Emission pattern of surface-enhanced Raman scattering from single nanoparticle-film junction," Applied Physics Letters, vol. 102, no. 8, p. 081117, 2013.

[5] S. Orbons and A. Roberts, "Resonance and extraordinary transmission in annular aperture arrays," Optics express, vol. 14, no. 26, pp. 12623-12628, 2006.

[6] K. K. Koerkamp, S. Enoch, F. B. Segerink, N. Van Hulst, and L. Kuipers, "Strong influence of hole shape on extraordinary transmission through periodic arrays of subwavelength holes," Physical review letters, vol. 92 , no. 18 , p. 183901, 2004.

[7] R. Gordon, A. Brolo, A. McKinnon, A. Rajora, B. Leathem, and K. Kavanagh, "Strong polarization in the optical transmission through elliptical nanohole arrays," Physical review letters, vol. 92, no. 3, p. 037401, 2004.

[8] B. Ni, L. Huang, J. Ding, G. Li, X. Chen, and W. Lu, "The collective property of enhanced transmission through compound metal periodic arrays of subwavelength apertures," Optics Communications, vol. 298, pp. 237-241, 2013.

[9] X. Zhang et al., "Tunable extraordinary optical transmission in a metal film perforated with two-level subwavelength cylindrical holes," Plasmonics, vol. 9, no. 5, pp. 1149-1153, 2014.

[10] S. G. Rodrigo et al., "Holes with very acute angles: a new paradigm of extraordinary optical transmission through strongly localized modes," Optics express, vol. 18, no. 23, pp. 23691-23697, 2010.

[11] J. Parsons, E. Hendry, C. P. Burrows, B. Auguié, J. R. Sambles, and W. L. Barnes, "Localized surfaceplasmon resonances in periodic nondiffracting metallic nanoparticle and nanohole arrays," Physical Review $B$, vol. 79, no. 7, p. 073412, 2009.

[12] Z. Jian, L. Jian-jun, and Z. Jun-wu, "Tuning the dipolar plasmon hybridization of multishell metaldielectric nanostructure: gold nanosphere in a gold nanoshell," Plasmonics, vol. 6, no. 3, p. 527, 2011.

[13] X. Ni, Z. Liu, A. Boltasseva, and A. V. Kildishev, "The validation of the parallel three-dimensional solver for analysis of optical plasmonic bi-periodic multilayer nanostructures," Applied Physics A, vol. 100, no. 2, pp. 365-374, 2010. 
[14] G. V. Naik, V. M. Shalaev, and A. Boltasseva, "Alternative plasmonic materials: beyond gold and silver," Advanced Materials, vol. 25, no. 24, pp. 3264-3294, 2013.

[15] M. Gospodinova-Daltcheva, R. Arnaudov, and P. Philippov, "Energy propagation properties of Al-lossy lines in high-speed circuits on silicon substrate," in 1999 Symposium on High Performance Electron Devices for Microwave and Optoelectronic Applications. EDMO (Cat. No. 99TH8401), 1999, pp. 295-300: IEEE.

[16] J. Qian, Z. Chen, W. Wang, Y. Li, J. Xu, and Q. Sun, "Dual symmetry breaking in gold-silica-gold multilayer nanoshells," Plasmonics, vol. 9, no. 6, pp. 1361-1369, 2014.

[17] E. Prodan and C. Radloff, "Halas. NJ; Nordlander, P," Science, vol. 302, pp. 419-422, 2003.

[18] Y. Ekinci, H. H. Solak, and C. David, "Extraordinary optical transmission in the ultraviolet region through aluminum hole arrays," Optics letters, vol. 32, no. 2, pp. 172-174, 2007.

[19] M. Najiminaini, F. Vasefi, B. Kaminska, and J. J. Carson, "A three-dimensional plasmonic nanostructure with extraordinary optical transmission," Plasmonics, vol. 8, no. 2, pp. 217-224, 2013.

[20] Y. Wang, Y. Qin, and Z. Zhang, "Extraordinary optical transmission property of X-shaped plasmonic nanohole arrays," Plasmonics, vol. 9, no. 2, pp. 203-207, 2014.

[21] S. Ogawa, S. Fukushima, and M. Shimatani, "Extraordinary Optical Transmission by Hybrid PhononPlasmon Polaritons Using hBN Embedded in Plasmonic Nanoslits," vol. 11, no. 6, p. 1567, 2021.

[22] Y. Lin, Y. Han, C. Song, and Y. Deng, "Topologically optimized periodic resonant nanostructures for extraordinary optical transmission [Invited]," Optical Materials Express, vol. 11, no. 7, pp. 2153-2164, 2021/07/012021.

[23] S. Tajik and Z. J. J. o. A. P. Atlasbaf, "Investigating extraordinary optical transmission and sensing performance through periodic bilayer magneto-plasmonic structure," vol. 127, no. 2, p. 023102, 2020.

[24] J. Park, H. Lee, A. Gliserin, K. Kim, and S. J. P. Kim, "Spectral shifting in extraordinary optical transmission by polarization-dependent surface plasmon coupling," vol. 15, no. 2, pp. 489-494, 2020.

[25] M. Born and E. Wolf, "Book Review: Principles of optics.-7th expanded ed./Cambridge U Press, 1999," The Observatory, vol. 120, p. 149, 2000.

[26] H. Schouten et al., "GW "t Hooft, D. Lenstra, and ER Eliel," Phys. Rev. Lett, vol. 94, p. $053901,2005$. 
[27] Y. H. Fu, J. B. Zhang, Y. F. Yu, and B. Luk'yanchuk, "Generating and manipulating higher order Fano resonances in dual-disk ring plasmonic nanostructures," ACS nano, vol. 6, no. 6, pp. 5130-5137, 2012.

[28] X. Zhang et al., "Enhanced optical transmission in a plasmonic nanostructure perforated with compound holes and nanorods," vol. 325, pp. 105-110, 2014.

[29] A. Degiron and T. W. Ebbesen, "Analysis of the transmission process through single apertures surrounded by periodic corrugations," Optics Express, vol. 12, no. 16, pp. 3694-3700, 2004/08/09 2004.

[30] Z. Zhang, F. Shi, and Y. J. P. Chen, "Tunable multichannel plasmonic filter based on coupling-induced mode splitting," vol. 10, no. 1, pp. 139-144, 2015.

[31] J. Wang, Y. Niu, D. Liu, Z.-D. Hu, T. Sang, and S. J. P. Gao, "Tunable plasmon-induced transparency effect in MIM side-coupled isosceles trapezoid cavities system," vol. 13, no. 2, pp. 609-616, 2018.

[32] M. R. Rakhshani, M. A. J. S. Mansouri-Birjandi, and A. B. Chemical, "High sensitivity plasmonic refractive index sensing and its application for human blood group identification," vol. 249, pp. 168-176, 2017.

[33] M. R. Rakhshani and M. A. J. P. Mansouri-Birjandi, "Utilizing the metallic nano-rods in hexagonal configuration to enhance sensitivity of the plasmonic racetrack resonator in sensing application," vol. 12, no. 4, pp. 999-1006, 2017.

[34] S. Khani, M. Danaie, and P. J. I. O. Rezaei, "Tunable single-mode bandpass filter based on metalinsulator-metal plasmonic coupled U-shaped cavities," vol. 13, no. 4, pp. 161-171, 2019.

[35] M. R. Rakhshani, A. Tavousi, and M. A. J. A. o. Mansouri-Birjandi, "Design of a plasmonic sensor based on a square array of nanorods and two slot cavities with a high figure of merit for glucose concentration monitoring," vol. 57, no. 27, pp. 7798-7804, 2018.

[36] A. Shabani, M. R. Roknabadi, M. Behdani, M. K. Nezhad, and N. J. J. o. N. Rahmani, "Extraordinary optical transmission of periodic array of subwavelength holes within titanium nitride thin film," vol. 11, no. 3, p. 036006, 2017.

[37] Y. Hu et al., "Extraordinary optical transmission in metallic nanostructures with a plasmonic nanohole array of two connected slot antennas," vol. 10, no. 2, pp. 483-488, 2015.

[38] M. Wang, M. Cao, X. Chen, and N. Gu, "Subradiant plasmon modes in multilayer metal-dielectric nanoshells," The Journal of Physical Chemistry C, vol. 115, no. 43, pp. 20920-20925, 2011. 
[39] P. Nordlander, C. Oubre, E. Prodan, K. Li, and M. Stockman, "Plasmon hybridization in nanoparticle dimers," Nano letters, vol. 4, no. 5, pp. 899-903, 2004.

[40] M. Bora et al., "Plasmon resonant cavities in vertical nanowire arrays," Nano letters, vol. 10, no. 8, pp. 2832-2837, 2010. 